\title{
REZENSIONEN
}

\section{Plädoyer für Verantwortung im Umgang mit der parlamentarischen Demokratie}

\author{
Niehuis, Edith: Die Demokratiekiller. Fehlentwicklungen in der deutschen Politik, Verlag \\ Lehmanns Media, Berlin 2011, 199 Seiten, € 17,95.
}

Der Titel klingt ein wenig reißerisch und ist wohl dem Versuch geschuldet, Aufmerksamkeit zu provozieren für ein Thema, das nicht nur der Autorin am Herzen liegt. Die langjährige Abgeordnete des Deutschen Bundestages, Parlamentarische Staatssekretärin in der ersten rot-grünen Regierung und Lehrbeauftragte an der FU Berlin verarbeitet hier Erfahrungen und Schlussfolgerungen aus der unmittelbaren politischen Arbeit, die sie kritisch reflektiert. Entstanden ist keine leidenschaftslose fachwissenschaftliche Analyse, sondern ein leidenschaftliches, fachlich gut fundiertes Plädoyer für Vernunft und Verantwortung beim Umgang mit den Handlungsbedingungen der parlamentarischen Demokratie.

Wer etwa von dem Untertitel „Fehlentwicklungen“ eine herkömmliche Auflistung gängiger Kritikpunkte oder eine nachträgliche Abrechnung mit allerlei Aporien und Restriktionen des politischen Geschäfts erwartet, sieht sich alsbald getäuscht. Die Autorin zeigt im Gegenteil keinerlei Verständnis für Politiker, die nach dem Ausscheiden aus der aktiven Rolle anfangen, über den Wassern zu schweben, „überparteilich“ zu werden und die Grundvoraussetzungen ihrer eigenen langjährigen Funktionen zu relativieren. Da schont sie niemanden, weder den Bundespräsidenten Richard von Weizsäcker, wenn er nach jahrzehntelanger Karriere in der CDU schließlich im höchsten Staatsamt die „Machtversessenheit“ und „Machtvergessenheit“ der Politiker entdeckt und Wohlwollen für die Wahlabstinenz von Bürgern zu erkennen gibt (S. 42 mit Fußnote 48), noch den Kanzler und Altkanzler Gerhard Schröder, wenn er mit der Formel „Erst das Land, dann die Partei“ den Beifall der Massenmedien freischaltet (S. 29 ff.)

Dieser Formel ist eines der Kapitel gewidmet, in denen der Reihe nach - Parteien, Medien, Bundestag, Lobby - die jeweiligen „Demokratiekiller“ identifiziert werden. Es handelt sich dabei überwiegend um Killerphrasen, die in der öffentlichen Diskussion ihre schädliche Wirkung tun: teils unbewusstes Nachbeten althergebrachter Redensarten, teils bewusster Einsatz von Formeln und Attitüden, die auf Zustimmung deshalb rechnen, weil sie Ressentiments gegen Parteien, Parlamente und „Berufspolitiker“ bedienen, die von weither kommen - von einheitsmythischen Restbeständen des Obrigkeitsstaats, von der Parteienprüderie der Weimarer Republik, von der Konfliktscheu und der Bequemlichkeit des umsorgten Konsumbürgers und von manchem mehr. Das Buch erweist sich als ein diskurskritisches Traktat, das dem Leser - dem politisch handelnden wie dem beobachtenden - vor Augen führen will, wo und warum die politische Sprache den Erfordernissen demokratischer Verhältnisse nicht angemessen ist und sie beschädigen kann.

Durch alle Kapitel zieht sich ein Querschnittsaspekt hindurch: Stellung, Aufgaben und Innenleben der politischen Parteien. Sie tauchen, und das mit Recht, überall auf, nicht nur in den ausdrücklich ihnen gewidmeten Abschnitten. Für den praktischen und politikwissenschaftlichen Kenner der Materie mag das selbstverständlich erscheinen, ist es doch genau die querschnittsartige Rolle, in der die Parteien mit ihrer Funktion der Identifizierung, 
Bündelung und programmatischen Konturierung der unzähligen Interessen, Erwartungen und Meinungen der pluralistischen Gesellschaft präsent zu sein haben. Jedoch muss man leider sagen: Dieser Ansatz ist angesichts der im öffentlich-medialen Diskurs grassierenden Parteienaversion geradezu mutig für eine Schrift, die in der Breite Gehör finden, die Leser von den Vorzügen einer institutionellen Ordnung überzeugen will, welche die Autorin ohne Scheu als „Parteiendemokratie“ bezeichnet - also als das, was sie nicht nur ist, sondern zu sein hat. Dass Parlamentarismus ohne Parteien nicht geht, weder historisch noch aktuell, kann jedermann leicht erkennen - dafür genügt schon ein Blick auf die innergesellschaftlichen Auseinandersetzungen in arabischen, afrikanischen und asiatischen Regionen -, aber nicht jeder spricht es so klar aus.

Die von Niehuis zusammengetragenen Beispiele für fahrlässiges Reden und sich wegduckendes Verhalten sind zahlreich, und sie zeigen die Hauptsorge der Autorin. Sie empfindet unsere Parteien als verunsichert, verzagt und auch verführt durch die dominante Rolle der Massenmedien und die davon ausgehende Versuchung, sich deren Eigeninteressen von Personalisierung, Skandalisierung und Simplifizierung anzupassen. Niehuis weist anhand umfangreichen empirischen Materials nach, dass die Unterwerfung unter vermeintlich unwiderstehliche Trends der Mediengesellschaft nicht nur den Parteien selbst nichts gebracht hat, sondern auch die Fähigkeit zu rationaler politischer Willensbildung, zur Rekrutierung politikfähigen Personals für Parlamentsmandate und Regierungsämter, schließlich zur glaubwürdigen und erfolgreichen Repräsentation gesellschaftlicher Interessen im staatlichen Handeln zerstören kann. Was sie dagegen fordert, ist eine andere Art der „Öffnung“ der Parteien als die derzeit meist diskutierte: Die Parteien sollen ihren Auftrag nicht an Medienprofis delegieren, sondern sich auf die Vernunft und Erfahrung ihrer immer noch zahlreichen engagierten Vertreter in Wahlkreisen und örtlichen Organisationen stützen; sie sollen dort auf alle Bürgerinitiativen und zivilgesellschaftlichen Mitwirkungsaktivitäten zugehen und sich beteiligen; sie sollen selbstbewusst ihre politisch-prozeduralen Fähigkeiten einbringen und so die Verbindung zwischen den sich differenzierenden Einzelinteressen und ihrer programmatischen Aufbereitung für die parlamentarische Repräsentation und die staatliche Entscheidungsfindung wiederherstellen. Nur so könne bürgerschaftliches Engagement, insbesondere auch jenes in den neuen technischen Kommunikationsformen des Internets, demokratisch bleiben.

Das ist nur eines, wohl aber das zentrale Anliegen dieser Schrift. Zahlreiche Analysen nebst daraus abgeleiteten Empfehlungen befassen sich mit den Arbeitsformen des Bundestages, mit der modernen Entwicklung von Lobbyarbeit und vielem mehr. Man muss nicht jeder Einschätzung der Autorin zustimmen, aber es lohnt sich überall, sie zur Kenntnis zu nehmen und zu prüfen. Und das Wichtigste: Es wäre zu wünschen, dass mehr Politiker so klar und selbstbewusst zum demokratischen Parlamentarismus in seiner gegenwärtigen Lage Stellung nehmen würden. Denn er kann nicht nur „gekillt" werden - die historische Erfahrung hat bewiesen, dass man ihn auch absterben lassen kann. Und das wäre wohl das Letzte, was Deutschland geschehen lassen darf angesichts einer Welt, in der täglich Blut vergossen wird im Kampf um die demokratischen Errungenschaften, derer wir uns so sicher wähnen. 